\title{
Aortic Dissection as a Complication in Patients on Maintenance Haemodialysis: Two Cases Report
}

\author{
Zhuo-Tao Xiao ${ }^{1}$, Xin-Zhong Huang ${ }^{1}$, Jian-Hua Wu ${ }^{1}$, Xia Jiang ${ }^{2 *}$, Xi Huang ${ }^{3 *}$ and Li Yuan ${ }^{*}$ \\ ${ }^{1}$ Division of Nephrology, Affiliated Hospital of Nantong University, Nantong, Jiangsu, 226001, China \\ ${ }^{2}$ Division of Nephrology, The Second Peoples Hospital of NanTong, Nantong, Jiangsu, 226001, China \\ ${ }^{3}$ School of Mechanical Engineering, Nantong University, Nantong, Jiangsu, 226019, China
}

*Corresponding author: Xia Jiang, Division of Nephrology, The Second Peoples Hospital of NanTong, Nantong, Jiangsu Province, 226001, China, Tel: 86513- 85169100; Fax: 86513- 85169100; E-mail: 846991044@qq.com

Xi Huang, School of Mechanical Engineering, Nantong University, Nantong, Jiangsu, 226019, China, Tel: 86513- 85012673; Fax: 86513- 85012671; Email: 47293245@qq.com

Li Yuan, Division of Nephrology, Affiliated Hospital of Nantong University, 20 Xisi Road, Nantong, Jiangsu Province, 226001, China, Tel: 8651385050212; Fax: 86513- 85106566; E-mail: yuanlint@163.com

Received date: May 19, 2016; Accepted date: June 27, 2017; Published date: July 05, 2017

Citation: Xiao ZT, Huang XZ, Wu JH, Jiang X, Huang X, et al. (2017) Aortic Dissection as a Complication in Patients on Maintenance Hemodialysis: Two Cases Report. J Clin Exp Nephrol Vol 2 Iss 3: 41.

Copyright: (c) 2017 Xiao ZT, et al. This is an open-access article distributed under the terms of the Creative Commons Attribution License, which permits unrestricted use, distribution, and reproduction in any medium, provided the original author and source are credited.

\section{Abstract}

Aortic dissection $(A D)$ is a rare disease that may result in a fatal outcome. Uremic patients are often associated with factors susceptible to $A D$. We herein report two cases of $A D$ occurring in maintenance haemodialysis patients. The two male Chinese had history of smoking and poor uncontrolled hypertension for many years. One patient died of the aortic wall rupture with conservative treatment. The other patient had a history of myocardial infarction and long-term administration of anti-platelet drugs. The patient survives after prompt stenting and continuous maintenance haemodialysis. It is important to improve our understanding about aortic dissection (AD) in haemodialysis patients because of the high mortality and morbidity. Once a patient on maintenance haemodialysis is diagnosed with $A D$, surgery or endovascular graft exclusion should be considered according to the actual situation of individual patients, and the mode of dialysis should be converted in conservation treatment patients.

Keywords: Aortic dissection; Haemodialysis; Endovascular graft exclusion

\section{Introduction}

Aortic dissection (AD) refers to the intimal tear due to damage of the normal structure of the aortic wall from various reasons. Mechanically, it has been considered that circulating blood flows into the media of the aorta through the rupture of the intima forming true and false lumens. It can extend and dissect, causing a high mortality rate of $60-70 \%$ within a week, and $90 \%$ within three months [1]. Uremic patients with chronic renal dysfunction are often associated with refractory hypertension, atherosclerosis and lipid metabolism disorders. These complications together with great fluctuations of blood volume before and after haemodialysis constitute risk factors of $A D$ in patients on maintenance haemodialysis. This article reports two cases of $A D$ occurring in patients on maintenance haemodialysis between 2000 and 2013 in the department of haemodialysis of the hospital.

\section{Case 1}

A 50-year-old Chinese male patient who had been on maintenance haemodialysis for a year at twice-a-week regular intervals came to the hospital because of sudden onset of persistent severe chest pain, when BP was 176/90 $\mathrm{mmHg}$ and HR was $88 \mathrm{bpm}$. He had a 20-year history of smoking and a 5year history of hypertension without satisfied control. His usual BP was higher than $160 / 100 \mathrm{mmHg}$. Admission CT scan showed dilation of the aortic arch and thoracic aorta. Contrast-enhanced CT scan demonstrated the existence of a linear high-intensity shadow in the aortic arch, descending aorta, abdominal aorta, bilateral common iliac arteries and branches. A diagnosis of Debakey III AD was made. The break was located in the aortic arch (Figures 1 and 2). Electrocardiography (ECG) showed LV high voltage and ST-T change. Chest X-ray showed aortic sclerosis, enlargement of the heart profile and widening of the mediastinum. Intravenous nitroglycerin was administered to maintain BP between 131 118/81 68 $\mathrm{mmHg}$. The patient was advised to take absolute bed-confinement and oxygen inhalation. At the same time heparin-free haemodialysis was prescribed. Surgical or intervention treatment was recommended but the patient refused and died of $A D$ rupture in a week. 


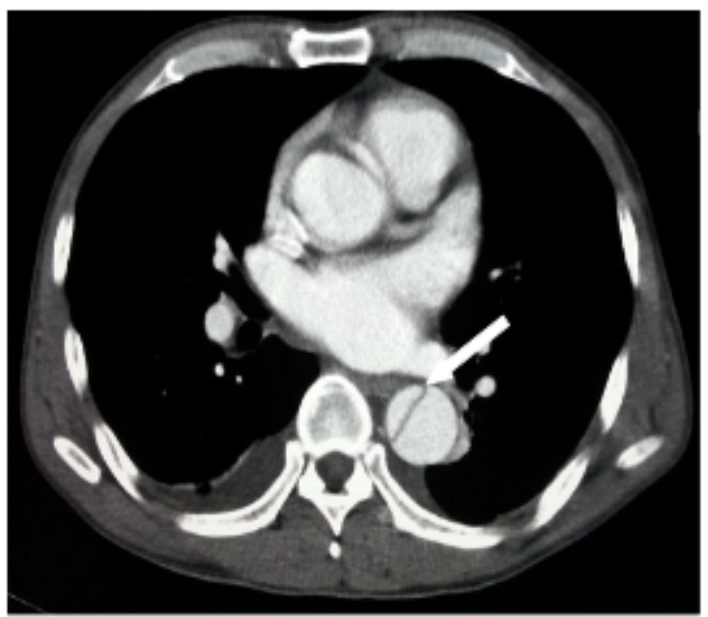

Figure 1: CT scan: aortic dissection (arrow).

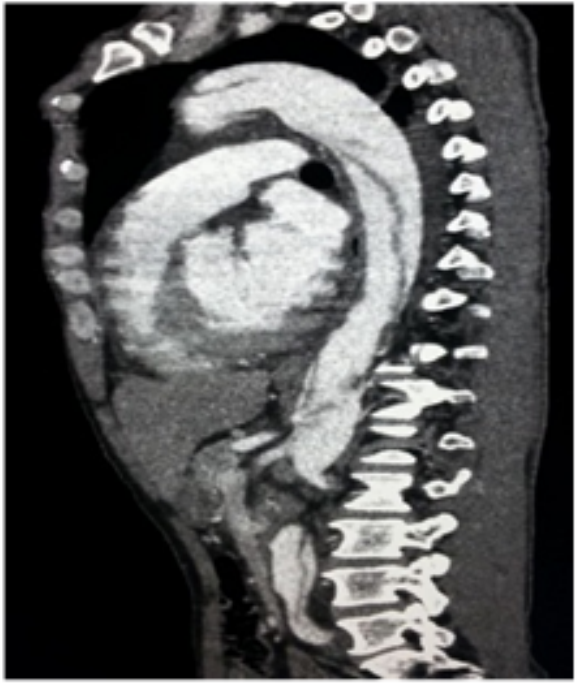

Figure 2: CT 3D imaging: aortic dissection.

\section{Case 2}

A 70-year-old Chinese male who had been on haemodialysis for 8 months at thrice-a-week regular intervals came to the hospital because of sudden onset of severe tearing chest pain. He had a 20-year history of smoking and a 15-year history of hypertension. Hypertension was not well-controlled with the maximum systolic pressure reaching $200 \mathrm{mmHg}$. He also had history of myocardial infarction 2 years ago and used aspirin routinely. Chest $\mathrm{X}$-ray suggested widening of the mediastinum. Contrast-enhanced CT scan suggested thickening of the aortic arch and thoracic aorta, scattered calcifications on the vascular wall, and an intimal flap moving medially in the lumen forming a true and a false double cavity. Partial contrast filling was observed in the false cavity. The diagnosis of DebakeyllI AD was made (Figure $\mathbf{3}$ ).

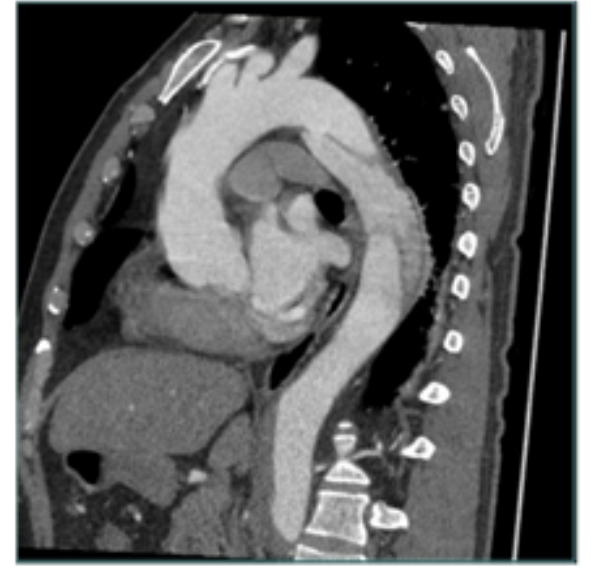

Figure 3: CT 3D imaging: aortic dissection.

After admission, the patient was administered Dolantin for analgesia and intravenous nitroglycerin to maintain BP. Heparinfree haemodialysis was performed. Endovascular graft exclusion was performed, and covered stents were implanted in the aortic arch and the thoracic aorta (Figure 4). Postoperative treatment included intensified BP control, maintenance haemodialysis using low-molecular-weight heparin for anti-coagulation, and administration of anti-platelet drugs. The patient has been followed up for three years, showing a stable general condition.

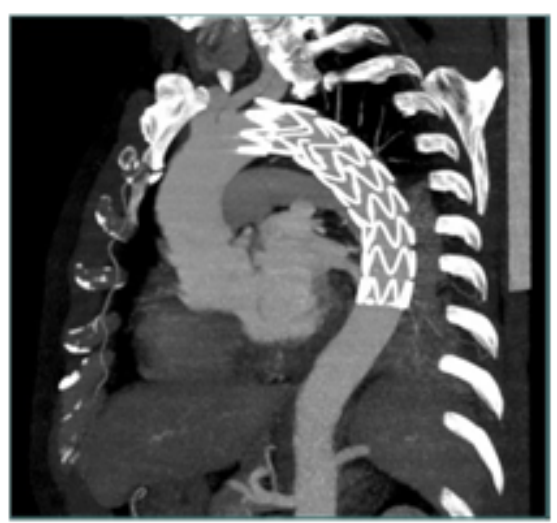

Figure 4: CT 3D imaging: post stent implantation.

\section{Discussion}

Aortic dissection is a rare but serious and life-threatening clinical condition in which blood flows into the damaged aortic wall. It is reported that tearing of the aortic wall does not stop until the eventual development of a fatal rupture in more than $60 \%$ acute $A D$ cases [2]. Patients on maintenance haemodialysis are often associated with hypertension, lipid and calcium phosphorus metabolic disorders, arteriosclerosis, and hemodynamic change, all of which may contribute to the occurrence of $A D$ [3-5]. Takeda et al. [6] investigated the cause of death in 113 chronic dialysis patients who died during a 10year follow-up period. Their autopsy study revealed that 5 
(5.4\%) of these patients died of $A D$, which is high than $0.59 \%$ in general populations.

Once the diagnosis of $A D$ is confirmed, appropriate conservative treatment is the first consideration before further treatment is instituted. Conservative treatment mainly includes lowering the LV systolic pressure to $110 \mathrm{mmHg}$, reducing $\mathrm{HR}$ and analgesia [7]. In De Bakey I or II AD, surgical resection of the ascending aorta is standard management. For De Bakey III AD, medical conservative treatment or intervention therapy is an alternative option [2]. Bleeding from the ruptured $A D$ is the severe complication of AD. Zheng et al. [8] reported an $A D$ patients presenting with myocardial ischemia was misdiagnosed as myocardial infarction and died of $A D$ rupture after administration of anti-coagulants and thrombolytic therapy. Jing et al. [9] observed 13 AD patients with co-existence of coronary artery diseases who underwent endovascular graft exclusion followed by use of anticoagulants during percutaneous transluminal intervention (PCl) and subsequent long-term administration of anti-platelet therapy after surgery. Their 11month follow-up study showed that anticoagulants and thrombolytic therapy were safe in patients who underwent endovascular stent graft exclusion. There is no guideline about how to manage in HD patients diagnosed with AD. Ouniss et al. [10] reported conservative treatment and conversion from haemodialysis to peritoneal dialysis in three haemodialysis patients when they developed $A D$, thus avoiding the use of anticoagulants during haemodialysis and reducing the impact of haemodialysis-related hemodynamic change on the aorta. No $A D$ rupture occurred during the surviving period of the patient after conversion to peritoneal dialysis. Both patients reported herein had a long history of hypertension, poor BP control, arteriosclerosis, vascular calcification, and smoking, all of which are susceptible factors to AD. The patient in Case 1 refused to receive surgical or intervention treatment after confirmation of the diagnosis and died of AD rupture in a week. The patient in Case 2 had a history of myocardial infarction and long-term aspirin administration. Stenting was performed after confirmation of the diagnosis. He is still maintained on haemodialysis and receives $100 \mathrm{mg}$ daily aspirin. His general condition has been good so far.

In summary, haemodialysis patients are often associated with various susceptible factors of $A D$, in whom the incidence of $A D$ is higher than that in general populations. If a haemodialysis patient suddenly complains of severe and persistent chest pain, $A D$ should be highly suspected. For haemodialysis patients with confirmed diagnosis of $A D$, surgery or endovascular stent graft exclusion should be considered, especially for the patients who didn't want to change the dialysis mode. As in maintained haemodialysis, hemodynamic changes and use of anticoagulants are likely to lead to deterioration and poor prognosis of AD. For those who choice conservative treatment, conversion to peritoneal dialysis should be considered.

\section{Conflict of Interest}

No conflict of interest was declared by the authors.

\section{Financial Support}

This work is supported by the peak of six personnel in Jiangsu Province. Jiangsu Government Scholarship (Grant No. JS-2014K004). Study abroad fund of Jiangsu Province.

\section{References}

1. Kato N, Shimono T, Hirano T, Suzuki T, Ishida M, et al. (2002) Midterm results of stent-graft repair of acute and chronic aortic dissection with descending tear: the complication-specific approach. J Thorac Cardiovasc Surg 124: 306-312.

2. Golledge J, Eagle KA (2008) Acute aortic dissection. Lancet 372: 55-66.

3. Jaradat MI, Molitoris BA (2002) Cardiovascular disease in patients with chronic kidney disease. Semin Nephrol 22: 459-473.

4. Nienaber CA, Eagle KA (2003) Aortic dissection: new frontiers in diagnosis and management: Part I: from etiology to diagnostic strategies. Circulation 108: 628-635.

5. Peev V, Nayer A, Contreras G (2014) Dyslipidemia, malnutrition, inflammation, cardiovascular disease and mortality in chronic kidney disease. Curr Opin Lipidol 25: 54-60.

6. Takeda K, Harada A, Okuda S, Fujimi S, Oh Y, et al. (1997) Sudden death in chronic dialysis patients. Nephrol Dial Transplant 12: 952-955.

7. Nienaber CA, Eagle KA (2003) Aortic dissection: new frontiers in diagnosis and management: Part II: therapeutic management and follow-up. Circulation 108: 772-778.

8. Zheng Z, Ye Z, Huang $Y, X u$ J, Cai R, et al. (2013) A case report of acute myocardial infarction concomitant with Standford type $B$ aortic dissection. J Cardiovasc Dis Res 4: 251-253.

9. Jing Q, Guo L, Wang X, Luan B, Wang G, et al. (2013) Percutaneous transluminal intervention and antiplatelet therapy following endovascular graft exclusion for Stanford B thoracic aortic dissection. Int J Cardiol 165: 478-482.

10. Ounissi M, Goucha R, Hedri H, Hamida FB, Abderrahim E, et al. (2009) Dissecting aortic aneurysm in maintenance hemodialysis patients. Saudi J Kidney Dis Transpl 20: 1053-1056. 